Pacific Journal of Mathematics

THE OBSTRUCTION OF THE FORMAL MODULI SPACE IN 


\title{
THE OBSTRUCTION OF THE FORMAL MODULI SPACE IN THE NEGATIVELY GRADED CASE
}

\author{
MARIE A. VITULLI
}

Consider a semigroup ring $B_{H}=k\left[t^{h} / h \in H\right]$ where $t$ is a transcendental over an algebraically closed field $k$ of characteristic 0 . Let $T^{1}(B)$ denote $T^{1}(B / k, B)$ where $T^{1}(B / k,-)$ is the upper cotangent functor of Lichtenbaum and Schlessinger. Then $T^{1}(B)$ is a graded $k$-vector space of finite dimension and $B$ is said to be negatively graded if $T^{1}(B)_{+}=$ 0 . It is known that a versal deformation $T / S$ of $B / k$ exists in the sense of Schlessinger, where $\left(S, m_{S}\right)$ is a complete noetherian local $k$-algebra. We say that the formal moduli space is unobstructed if $S$ is a regular local ring. In this paper we restrict our attention to the negatively graded semigroup rings. In this case we compute the dimension of $T^{1}(B)$ and are thus able to determine which formal moduli spaces are unobstructed.

Let $U$ denote the (open) subset of $\operatorname{Spec}(S)$ consisting of all points with smooth fibres. In a previous paper [5] we computed the dimension of $U$. We always have inequalities:

$$
\operatorname{dim} U \leqq\left(\text { Krull) } \operatorname{dim} S \leqq\left[m_{S} / m_{S}^{2}: k\right]\right. \text {. }
$$

Consequently $S$ is a regular local ring if and only if $\operatorname{dim} U=\left[m_{S} /\right.$ $\left.m_{S}^{2}: k\right]=\left[T^{1}(B): k\right]$. In the general case the difference $\left[T^{1}(B): k\right]-$ $\operatorname{dim} U$ gives some indication of the extent of the obstruction.

I would like to express my gratitude to Dock S. Rim for stimulating my interest in the subject and for his valuable suggestions and advice.

\section{Preliminaries and notation.}

(2.1) Let $H$ be a subsemigroup of the additive subgroup $N$ of nonnegative integers. $H$ is called a numerical semigroup if the greatest common divisior of the elements of $H$ is 1 , so that only finitely many positive integers are missing from $H$. Such elements are called the gaps of $H$ and the number of gaps is called the genus of $H$, denoted by $g(H)$. The least positive integer $c$ such that $c+N \subset H$ is called the conductor of $H$, denoted by $c(H)$. The least positive integer $m$ in $H$ is called the multiplicity of $H$ and is denoted by $m(H)$. Throughout this paper $H$ will denote a numerical semigroup, $k$ an algebraically closed field of characteristic 0 . 
Let $B_{H}$ denote the $k$-subalgebra of the polynomial ring $k[t]$ generated by the monomials $t^{h}, h \in H . \quad B_{H}$ is called the semigroup ring of $H$.

When no possible confusion can arise we simply write $B$ for $B_{H}, g$ for $g(H), c$ for $c(H)$ and $m$ for $m(H)$.

(2.2) We now construct a generating set called the standard basis for $H$, denoted $S_{H}$. Let $m=m(H)$. For $0 \leqq j \leqq m-1$ choose $a_{j}$ to be the least positive integer in $H$ such that $a_{j} \equiv j$ $(\bmod m)$.

For $1 \leqq j \leqq k \leqq m-1$, set

$$
f_{j, k}=X_{j} X_{k}-X_{0}^{e(j, k)} X_{r(j, k)}
$$

where $0 \leqq r(j, k) \leqq m-1$ and $a_{j}+a_{k}=e(j, k) m+a_{r(j, k)}$. Set $I=$ $I_{H}$ equal to the ideal of $P=k\left[X_{0}, \cdots, X_{m-1}\right]$ generated by $\left\{f_{j, k}\right\}_{1 \leqq j \leqq k \leqq m-1}$ where $P$ is a polynomial algebra over $k$.

Proposition 2.3. If we define a k-algebra map $\varphi: k\left[X_{0}, \cdots, X_{m-1}\right] \rightarrow$ $B$ by $\varphi\left(X_{j}\right)=t^{a_{j}}$ for $0 \leqq j \leqq m-1$ then $0 \rightarrow I \rightarrow P \rightarrow B \rightarrow 0$ is exact. Furthermore, if we assign the weight $a_{j}$ to $X_{j}$ in $P$, then $\varphi$ is a homomorphism (of degree 0 ) of graded k-algebras and $I$ is homogeneous.

We will not attempt to give a precise definition of $T^{*}$ here. For definition and details of $T^{0}, T^{1}$ one can consult [1]; for the full cohomological properties one should consult Rim's article "Formal Deformation Theory" [4] (note that our $T^{i}$ plays the role of Rim's $D^{i}$ ). We state here some properties of $T^{*}$ that will facilitate our computations. For proofs of these assertions see [4] and [5].

Proposition 2.4. Let $P$ be a polynomial algebra over $R$ and let $0 \rightarrow I \rightarrow P \rightarrow A \rightarrow 0$ be exact. Then for any $A$-module $M$,

$$
\begin{aligned}
T^{\circ}(A \mid R, M) \cong & \operatorname{Der}_{R}(A, M), \\
T^{1}(A \mid R, M) \cong & \operatorname{Coker}\left(\operatorname{Der}_{R}(P, M) \longrightarrow \operatorname{Hom}_{A}\left(I / I^{2}, M\right)\right) \\
\cong & \text { the set of isomorphism classes of } R \text {-algebra } \\
& \text { extensions of } A \text { by } M .
\end{aligned}
$$

(2.5) In our case, if $B=B_{H}$ then $T^{1}(B)=T^{1}(B \mid k, B)$ becomes a graded $k$-vector space via the exact sequence of (2.3). We then have

$$
\begin{aligned}
T^{1}(B) & =\bigoplus_{-\infty<p<\infty} T^{1}(B)_{p} \\
& \cong \bigoplus_{-\infty<p<\infty} \operatorname{Coker}\left(\operatorname{Der}_{k}(P, B)_{p} \longrightarrow \operatorname{Hom}_{B}\left(I / I^{2}, B\right)_{p}\right)
\end{aligned}
$$


so that

$$
\begin{array}{r}
\left.T^{1}(B)_{p} \cong \text { the set of isomorphism classes of (degree } 0\right) \\
\text { graded } k \text {-algebra extensions of } B \text { by } B(p)
\end{array}
$$

where $B(p)$ is the graded $k$-module obtained from $B$ by shifting the degree by $p$; i.e., $B(p)_{n}=B_{p+n}$.

Those monomial curves $B_{H}$ for which $T^{1}(H)_{+}=T^{1}\left(B_{H}\right)_{+}=0$ are the so called negatively graded semigroup rings of Pinkham [3]. In [5] we completely classified these and described a method for computing $T^{1}(H)_{p}$. We now recall these results and set up some notation which will be used in $\S 3$.

(2.6) Let $S_{H}=\left\{a_{0}=m, a_{1}, \cdots, a_{m-1}\right\}$ denote the standard basis for $H$ (as in 2.2). For each integer $p$ let $G_{p}=\left\{a \in S_{H} \mid a+p \notin H\right\}$ and let $R_{p}=\left\{f_{j, k} \in I_{H} \mid a_{j}+a_{k}+p \notin H\right\}$. By abuse of notation associate with each $f_{j, k}$ of $R_{p}$ a vector $f_{j, k}=\left(f_{j, k}^{0}, \cdots, f_{j, k}^{m-1}\right)$ of $k^{m}$ where the $l$ th component is given by

$$
\begin{array}{rlrl}
f_{j, k}^{l}=-e(j, k) & & \text { if } l=0 \text { and } r(j, k) \neq 0, \\
& =-(e(j, k)+1) & & \text { if } l=0=r(j, k), \\
& =-1 & & \text { if } l=r(j, k) \neq 0, \\
& =2 & & \text { if } l=j=k, \\
& =1 & & \text { if } l=j \text { or } l=k \text { and } j \neq k, \\
& =0 & & \text { otherwise. }
\end{array}
$$

Again by abuse, let $R_{p}$ denote the vector subspace of $k^{m}$ spanned by those $f_{j, k}$ in $R_{p}$. We note that if $a_{l} \notin G_{p}$ then $f_{j, l}^{l}=0$ for all $f_{j, k} \in R_{p}$. Thus if $G_{p} \neq \varnothing, \operatorname{dim} R_{p} \leqq \# G_{p}-1$.

Proposition 2.7. In the notation above,

$$
\operatorname{dim} T_{p}=\operatorname{dim} T^{1}(H)_{p}=\max \left\{0, \# G_{p}-\operatorname{dim} R_{p}-1\right\} .
$$

(2.8) We say that $H$ is an ordinary semigroup of multiplicity $m$, denoted by $H_{m}$, if $H=\{0, m, m+1, m+2, \cdots\}$. We say that $H$ is hyperordinary if $H=m N+H_{m^{\prime}}$ where $H_{m^{\prime}}$ is ordinary and $0<m<m^{\prime}$.

THEOREM 2.9. $H$ is negatively graded if and only if $H$ is of one of the following types:

(i) $H$ is ordinary;

(ii) $H$ is hyperordinary;

(iii) Excluding the above two cases, $H$ is negatively graded of multiplicity $m$ if and only if there exists precisely one gap $m+i$ 
between $m$ and $2 m$; if $i=1$ then $2 m+1 \notin H$ (or $H$ would be hyperordinary).

$$
\begin{aligned}
& \text { If } 2 \leqq i \leqq m-1 \text { then } \\
& H_{m, i}=\{0, m, m+1, \cdots, \widehat{m+i}, m+i+1, m+i+2, \cdots\} .
\end{aligned}
$$

If $i=1$ we have

$$
H_{m, 1}=\{0, m, m+2, \cdots, 2 m, 2 m \widehat{+1}, 2 m+2,2 m+3, \cdots\} .
$$

3. A Dimension formula for $T^{1}(H)$. We now compute the dimension of the tangent space $T^{1}(H)$ for the negatively graded semigroup rings. We first deal with the ordinary and hyperordinary cases and finally with those of the third type.

For these semigroups $T^{1}(H)=T^{1}\left(H_{-}\right)$. Recall the notation of (2.6) and let $a=a(H)$ denote the least positive integer in $H$ $m(H) N$, let $c=c(H)$. Then $p \leqq 2 a-c$ entails $R_{-p}=\varnothing$ since for $f_{j, k} \in I$ we have $a_{j}+a_{k}-p \geqq 2 a-p \geqq c$ so that $a_{j}+a_{k}-p \in H$. Thus by Proposition $2.7 \operatorname{dim} T^{1}(H)_{-p}=\max \left\{0, \sharp G_{-p}-1\right\}$.

Throughout these computations $[r]=$ the greatest integer $\leqq r$; $\{r\}=$ the least integer $\geqq r ; \delta_{r, s}$ denotes the Kronecker delta, i.e., $\delta_{r, s}=1$ if $r=s$ and 0 otherwise. Once a semigroup $H$ is fixed we let $T_{-l}=T^{1}(H)_{-l}$. By $\operatorname{dim}()$ we mean dimension as a $k$-vector space.

Now assume $H$ is ordinary or hyperordinary so that $H=m N+$ $\{p m+i, p m+i+1, p m+i+2, \cdots\}$ where $p \geqq 1$ and $1 \leqq i \leqq m-$ 1. Then $a(H)=p m+i$.

Proposition 3.1. Let $H=m \boldsymbol{N}+\{p m+1, p m+2, \cdots\}$. Then

$$
\begin{array}{rlrl}
\operatorname{dim} T_{-l} & =l-1 & & \text { if } 1 \leqq l \leqq m-1, \\
& =m-2 & & \text { if } l=m \text { or } m+1 \leqq l \leqq p m+2 \\
& & \text { and } m \nmid l, \\
& =m-1 & & \text { if } m+1 \leqq l \leqq p m+2 \text { and } m \mid l, \\
& =(p+1) m-l+\delta_{l,(p+1) m} & & \text { if } p m+3 \leqq l \leqq(p+1) m, \\
& =\delta_{m, 2} & & \text { if }(p+2) m \leqq l \leqq(2 p+1) m \\
& =0 & & \text { and } m \mid l, \\
& \text { otherwise. }
\end{array}
$$

Consequently,

$$
\begin{aligned}
\operatorname{dim} T^{1}(H) & =(p-1)(m-1)^{2}+m(m-1)-1 & \text { if } m \geqq 3, \\
& =2 p & \text { if } m=2 .
\end{aligned}
$$

Proof. Note that $2 a(H)-c(H)=p m+2$ so that for $1 \leqq l \leqq$ 
$p m+2$ we have $\operatorname{dim} T_{-l}=\# G_{-l}-1$.

Suppose $l>(p+1) m$ and set $q=l-[l / m] m+\delta_{l,[l / m] m} m$. If $q=1$ then $R_{-l} \supseteqq\left\{f_{1,1}, \cdots, f_{1, m-1}\right\} ; \quad$ if $q=2 \leqq m-1$ then $R_{-l} \supseteqq$ $\left\{f_{1,2}, \cdots, f_{1, m-1}, f_{2,2}\right\} ;$ if $3 \leqq q \leqq m$ then $R_{-l} \supseteqq\left\{f_{1,1}, \cdots, \hat{f}_{1, q-1}, \cdots, f_{1, m-1}\right.$, $\left.f_{2, q-1}\right\}$. Finally if $q=2=m$ we see that $R_{-l}=\varnothing$ for $2(p+2) \leqq l \leqq$ $2(2 p+1)$ while $R_{-l}=\left\{f_{1,1}\right\}$ for $l>2(2 p+1)$. Our assertions follow.

Then assume $p m+3 \leqq l \leqq(p+1) m$ and set $q=l-p m$ so that $G_{-l}=S_{H}-\left\{a_{q}\right\}$ if $q<m$ while $G_{-(p+1) m}=S_{H}$. Then $R_{-l}=$ $\left\{f_{j, k} \mid a_{j}+a_{k}<p m+a_{q}\right\}=\left\{f_{j, k} \mid j+k \leqq q-1\right\}$.

Set $R_{-l}^{\prime}=\left\{f_{1,1}, \cdots, f_{1, q-2}\right\}$. Then $R_{-l}^{\prime}$ generates $R_{-l}$ for if $j+k \leqq$ $q-1$ and $j \geqq 2$ we have (as vectors) $f_{j, k}=f_{1, j+k-1}+\cdots+f_{1, j}-$ $\left(f_{1, k-1}+\cdots+f_{1,1}\right)$. Since $\operatorname{rank} R_{-l}^{\prime}=q-2$ we have $\operatorname{dim} T_{-l}=(p+$ 1) $m-l+\delta_{l,(p+1) m}$.

Summing up the various components we see that

$$
\begin{aligned}
\operatorname{dim} T^{1}(H) & =(p-1)(m-1)^{2}+m(m-1)-1 & \text { if } m \geqq 3, \\
& =2 p & \text { if } m=2 .
\end{aligned}
$$

Now suppose $H=m N+\{p m+i, p m+i+1, \cdots\}$ where $2 \leqq$ $i \leqq m-1$. Then $c(H)=a(H)=p m+i=a_{i}$. We treat the cases $2 i \leqq m$ and $2 i>m$ separately but as the proofs are analagous we only give the former.

Proposition 3.2. Suppose that $H=m N+\{p m+i, p m+i+$ $+1, \cdots\}$ where $2 \leqq i \leqq m / 2$. Then

$$
\begin{aligned}
& \operatorname{dim} T^{1}(H)_{-l}=l \\
& =l-1 \\
& =l-2 \\
& =m-2 \\
& =m-1 \\
& =m-2(l-p m-i)-\delta_{l, p m+i+1} \\
& \text { if } 1 \leqq l \leqq i-1 \text {, } \\
& \text { if } i \leqq l \leqq m-i \text {, } \\
& \text { if } m-i+1 \leqq l \leqq m \text {, } \\
& \text { if } m+1 \leqq l \leqq p m+i \text { and } \\
& m \nmid l \text {, } \\
& \text { if } m+1 \leqq l \leqq p m+i \text { and } \\
& m \mid l \text {, } \\
& \text { if } p m+i+1 \leqq l \leqq p m \\
& +2 i-1 \text {, } \\
& =m-2(l-p m-i)+1+\delta_{l,(p+1) m} \\
& +\delta_{l,(p+1) m+1} \\
& \text { if } p m+2 i \leqq l \leqq p m \\
& +2 i+1 \text {, } \\
& =m-\min (2 i+1, m-1)-1 \quad \text { if } l=p m+2 i+2 \text {, } \\
& +\delta_{l,(p+1) m}+\delta_{i, 2} \\
& =(p+1) m-l+\delta_{l,(p+1) m} \\
& =0 \\
& \text { if } p m+2 i+3 \leqq l \\
& \leqq(p+1) m \text {, } \\
& \text { otherwise . }
\end{aligned}
$$


Consequently,

$\operatorname{dim} T^{1}(H)=(p-1)(m-1)^{2}+m(m-1)+i(i-2)+\delta_{i, 2}$.

Proof. Now $2 a(H)-c(H)=a(H)=p m+i$ so for $1 \leqq l \leqq p m+i$ we have $\operatorname{dim} T_{-l}=\# G_{-l}-1$.

For $p m+i+1 \leqq l \leqq(p+1) m+i-1$ we set $q=l-[l / m] m+$ $m \cdot \delta_{l,(p+1) m}$. Then $G_{-l}=S_{H}-\left\{a_{q}\right\}$ if $q \neq m$ and $G_{-(p+1) m}=S_{H}$. We note that $R_{-l}=\left\{f_{j, k} \mid a_{j}+a_{k}<a_{i}+l\right.$ and $\left.j+k \not \equiv q(\bmod m)\right\}$. Then $R_{-(p m+i+1)}=\left\{f_{i, i}\right\}$ entails $\operatorname{dim} T_{-(p m+i+1)}=m-3$.

Suppose that $p m+i+2 \leqq l \leqq p m+2 i-1$. Then $R_{-l}=$ $\left\{f_{j, k} \mid j+k \leqq i+q-1\right.$ and $\left.k \geqq j \geqq i\right\}$ and is generated by $R_{-l}^{\prime}=$ $\left\{f_{i, i}, \cdots, f_{i, q-1}, f_{i+1, i+1}, \cdots, f_{i+1, q-2}\right\}$. For suppose $f_{j, k} \in R_{-l}-R_{-l}^{\prime} \quad$ so that $j \geqq i+2, k \leqq q-3$. Then $i+2<j+k-i \leqq q-1$ and as vectors $f_{j, k}=\Delta_{j+k}-\Delta_{j}-\Delta_{k}$ where $\Delta_{r}=\sum_{s=i+1}^{r-i-1}\left(f_{i, s+1}-f_{i+1, s}\right)$.

As for independence, we observe that $f_{i, i}, \cdots, f_{i, m-1}, f_{i+1, i+1}, \cdots$, $f_{i+1,2 i-1}$ are independent. This is more readily seen by substituting the vectors

$$
v_{r}=f_{i, r+1}-f_{i+1, r} \text { if } i+1 \leqq r \leqq 2 i-2
$$

and

$$
\begin{aligned}
v_{2 i-1} & =f_{i, i}+f_{i, 2 i}-f_{i+1,2 i-1} \text { if } 2 i<m, \\
& =-f_{i+1,2 i-1} \text { if } 2 i=m
\end{aligned}
$$

for the last $i-1$ vectors.

Thus $\operatorname{dim} R_{-l}=2(l-p m-i)-2$ and $\operatorname{dim} T_{-l}=m-2(l-p m-$ i) for $p m+i+2 \leqq l \leqq p m+2 i-1$.

We wish to consider those integers $l$ between $p m+2 i$ and $(p+1) m+i-1$.

Suppose $p m+2 i \leqq l \leqq p m+2 i+1$ and let $q=l-p m$. Then $R_{-l}^{\prime}=\left\{f_{i, i}, \cdots, \hat{f}_{i, q-i}, \cdots, f_{i, \min (q-1, m-1)}, f_{i+1, i+1}, \cdots, f_{i+1, q-2}\right\} \quad$ generates $R_{-l}$ as above and has $\operatorname{rank} 2(q-i)-3-\delta_{l,(p+1) m+1}$.

Let $l=p m+2 i+2$ and set $q=2 i+2$. If $i=2$ so that $q=6$ then $R_{2-l}=\left\{f_{1,2}, f_{2,2}, f_{2,3}\right\} \quad$ if $m=4$ and $R_{-l}=\left\{f_{2,2}, f_{2,3}, \hat{f}_{2,4}, \cdots, f_{2}\right.$, $\left.\min (5, m-1), f_{3,4}\right\}$ if $m \geqq 5$. In either case rank $R_{-l}=\sharp R_{-l}-1$ as we note that

$$
\begin{array}{ll}
f_{1,2}=f_{2,2}-f_{2,3} & \text { if } m=4, \\
f_{3,4}=f_{2,3}-f_{2,2} & \text { if } m=5, \\
f_{3,4}=f_{2,3}-f_{2,2}+f_{2,5} & \text { if } m \geqq 6 .
\end{array}
$$

So we have $\operatorname{dim} R_{-l}=\min (q-1, m-1)-2+\delta_{m, 4^{*}}$. If $i \geqq 3$ then set $R_{-l}^{\prime}=\left\{f_{i, i}, \hat{f}_{i, i+1}, \cdots, f_{i, \min (q-1, m-1)}, f_{i+1, i+2}, \cdots, f_{i+1,2 i-1}, f_{i+2, i+2}\right\}$. Note that $\left(f_{i+1, i+1}-f_{i, i+2}\right)=f_{i+1, i+3}-f_{i+2, i+2}+f_{i+1, i+2}-f_{i, i+3}$ and if $2 i<m$ 
we have $f_{i+1,2 i}=f_{i, i+1}-f_{i, i}+\left(1-\delta_{2 i+1, m}\right) f_{i, 2 i+1}$. So $R_{-l}^{\prime}$ generates $R_{-l}$ as above and has rank $\min (q-1, m-1)-1$.

Now assume that $l>p m+2 i+2$. If $l \leqq(p+1) m$ set $q=l-$ $p m$ and let $R_{-l}^{\prime}=\left\{f_{i, i}, \cdots, \hat{f}_{i, q-i}, \cdots, f_{i, q-1}\right\} \cup B_{-l}$ where

$$
\begin{aligned}
B_{-l} & =\left\{f_{i+1, i+1}, \cdots, f_{i+1},{ }_{2 i-1}\right\} \text { if } q>3 i \\
& =\left\{f_{i+1}, i+1, \cdots, \hat{f}_{i+1, q-i-1}, \cdots, f_{i+1,2 i-1}, f_{i+2, q-i-1}\right\} \\
& \text { if } 2 i+3 \leqq q \leqq 3 i .
\end{aligned}
$$

Observe that if $f_{i+1, j} \in R_{-l}$ and $j \geqq 2 i$, setting $t=[j / i]$ we have $f_{i+1, j}=\left(1-\delta_{j, m-1}\right) f_{i, j+1}-\left[f_{i, j-i}+f_{i, j-2 i}+\cdots+f_{i, j-(t-1) i}\right]+\left[f_{i, j-i+1}+\right.$ $\left.f_{i, j-2 i+1}+\cdots+f_{i, j-(t-1) i+1}\right]+\left(1-\delta_{j, t i}\right)\left[f_{i+1, j-(t-1) i}-f_{i, j-(t-1) i+1}\right] . \quad$ Similarly if $i=2$ then $f_{i+2, q-i-1}=f_{4, q-3}$ is in the span of $R_{-l}^{\prime}$. Finally note that $\left(f_{i, q-i}-f_{i+1, q-i-1}\right)=\left(f_{i+1, q-i}-f_{i+2, q-i-1}\right)+\left(f_{i, i+2}-f_{i+1, i+1}\right)$ so that $R_{-l}^{\prime}$ generates $R_{-l}$ as above. Hence $\operatorname{dim} R_{-l}=q-2$.

If $(p+1) m+i-1 \geqq l>(p+1) m$ (and $l>p m+2 i+2)$ set $q=l-p m$ so that $i+3 \leqq q-i \leqq m-1$. Set

$$
R_{-l}^{\prime}=\left\{f_{i, i}, \cdots, \hat{f}_{i, q-i}, \cdots, f_{i, m-1}\right\} \cup B_{-l}
$$

where

$$
\begin{aligned}
B_{-l} & =\left\{f_{i+1, i+1}, \cdots, f_{i+1,2 i-1}\right\} \text { if } q>3 i, \\
& =\left\{f_{i+1, i+1}, \cdots, \hat{f}_{i+1, q-i-1}, \cdots, f_{i+1,2 i-1}, f_{i+2, q-i-1}\right\} \text { if } 2 i+3 \leqq q \leqq 3 i .
\end{aligned}
$$

Then $R_{-l}^{\prime}$ generates $R_{-l}$ as it has maximal rank $m-2$. Hence $T_{-l}=0$.

Finally suppose that $l \geqq(p+1) m+i$ (and $l>p m+2 i+2)$ and set $q=l-[l / m] m$. If $1 \leqq q \leqq i-1$ so that $l \geqq(p+2) m$ then

$$
R_{-l} \supseteqq\left\{f_{1,1}, \cdots, \hat{f}_{1, q-1}, \cdots, f_{1, m-1}, f_{2, q-1}\right\} \text {. }
$$

If $i \leqq q \leqq 2 i-1$ then

$$
R_{-l} \supseteqq\left\{f_{i, i}, \cdots, f_{i, m-1}, f_{i+1, i+1}, \cdots, f_{i+1,2 i-1}\right\} .
$$

If $2 i \leqq q \leqq m-1$ then

$$
R_{-l} \supseteqq\left\{f_{i, i}, \cdots, \hat{f}_{i, q-i}, \cdots, f_{i, m-1}\right\} \cup B_{-l}
$$

where

$$
\begin{aligned}
B_{-l}= & \left\{f_{i+1, i+1}, \cdots, f_{i+1,2 i-1}, f_{i+1, q}\right\} \text { if } q \leqq 2 i+1 \text { or } q>3 i, \\
= & \left\{f_{i+1, i+2}, \cdots, f_{i+1,2 i-1}, f_{i+1, q}, f_{i+2, i+2}\right\} \text { if } q=2 i+2, \\
= & \left\{f_{i+1, i+1}, \cdots, \widehat{f_{i+1, q-i-1}}, \cdots, f_{i+1,2 i-1}, f_{i+1, q}, f_{i+2, q-i-1}\right\} \\
& \text { if } 2 i+3 \leqq q \leqq 3 i .
\end{aligned}
$$

If $q=0$ so that $l \geqq(p+2) m$ then 


$$
R_{-l} \supseteqq\left\{f_{1,1}, \cdots, \widehat{f_{1, i-1}}, \cdots, \widehat{f_{1, m-1}}, f_{i, m-i+1}, f_{i+1, m-1}\right\} .
$$

In all cases $\operatorname{dim} R_{-l}=m-1$ so that $T_{-l}=0$.

Proposition 3.3. Suppose $H=m N+\{p m+i, p m+i+1, \cdots\}$ where $i \geqq 2$ and $2 i>m$. Then

$$
\begin{array}{rlrl}
\operatorname{dim} T^{1}(H)_{-l} & =l & & \text { if } 1 \leqq l \leqq m-i, \\
& =l-1 & & \text { if } m-i+1 \leqq l \leqq i-1, \\
& =l-2 & & \text { if } i \leqq l \leqq m, \\
& =m-2 & & \text { if } m+1 \leqq l \leqq p m+i \\
& =m-1 & & \text { and } m \nmid l, \\
& & \text { if } m+1 \leqq l \leqq p m+i \\
& =m-2(l-p m-i)-\delta_{l, p m+i+1}+\delta_{l, i p+1) m} \\
& & & \text { if } p m+i+1 \leqq l \leqq(p+1) m, \\
& =p m+2 i-l+\delta_{l,(p m+2 i)} & & \text { if }(p+1) m+1 \leqq l \leqq p m+2 i, \\
& =1 & & \text { if } l=p m+2 i+2 \text { and } i=2, \\
& =0 & & \text { otherwise. }
\end{array}
$$

Consequently, $\operatorname{dim} T^{1}(H)=(p-1)(m-1)^{2}+m(m-1)+i(i-2)+\delta_{i, 2}$.

CoRollaRY 3.4. Suppose $H$ is ordinary or hyperordinary of multiplicity $m$ and $a(H)=p m+i$. Then

$$
\begin{aligned}
\operatorname{dim} T^{1}(H) & =(p-1)(m-1)^{2}+m(m-1)+i(i-2)+\delta_{i, 2} \text { if } m \geqq 3, \\
& =2 p \text { if } m=2 .
\end{aligned}
$$

We finally deal with those negatively graded semigroups of the third type so that there is precisely one gap $m+i$ between $m$ and $2 m$. Recall that if $i=1$ then $2 m+1 \notin H$. In any case, $a_{j}=m+j$ for $j \neq i$ while $a_{i}=a_{j}+a_{k}$ whenever $j+k=i+\delta_{i, 1} m$. Again we deal with a series of cases governed by the relation of $i$ and $m$. As the proofs are similar we only give the proof in case $2 \leqq i \leqq m-$ $1 \leqq 2 i$.

Proposition 3.5. Let $H=H_{m}-\{m+1,2 m+1\}$ where $H_{m}$ is ordinary and $m \geqq 3$. Then

$$
\begin{aligned}
\operatorname{dim} T^{1}(H)_{-l} & =l-\left[\frac{l+1}{2}\right]+\delta_{l, 1} & & \text { if } 1 \leqq l \leqq m-2, \\
& =l-\left[\frac{l+1}{2}\right]-1 & & \text { if } m-1 \leqq l \leqq m+1,
\end{aligned}
$$




$$
\begin{aligned}
& =l-\left[\frac{l+1}{2}\right]-3+\delta_{l, m+2} \quad \text { if } m+2 \leqq l \leqq m+4 \\
& \text { and } l \leqq 2 m-2 \text {, } \\
& =m-\left[\frac{l+1}{2}\right]+\delta_{l, m+6} \quad \text { if } m+5 \leqq l \leqq 2 m-2 \text {, } \\
& =\delta_{m, 5}+\delta_{m, 7} \quad \text { if } l=2 m-1 \text {, } \\
& =1+\delta_{m, 4}+\delta_{m, 6} \quad \text { if } l=2 m \text {, } \\
& =\delta_{m, 3}+\delta_{m, 5} \quad \text { if } l=2 m+1 \text {, } \\
& =\delta_{m, 4} \\
& =\delta_{m, 3}+\delta_{m, 4} \\
& =\delta_{m, 3} \\
& =0 \\
& \text { if } l=2 m+2 \text { or } 3 m+2 \text {, } \\
& \text { if } l=3 \mathrm{~m} \text {, } \\
& \text { if } l=3 m+1,4 m \text { or } 5 m \text {, } \\
& \text { otherwise. }
\end{aligned}
$$

Consequently,

$$
\operatorname{dim} T^{1}(H)=\frac{m(m-1)}{2}+2+3 \delta_{m, 3}+2 \delta_{m, 4}
$$

Proposition 3.6. Suppose $H=H_{m}-\{m+i\}$ where $H_{m}$ is ordinary and $2 \leqq i \leqq(m-2) / 2$. Then

$$
\begin{array}{rlrl}
\operatorname{dim} T^{1}(H)_{-l} & =l & & \text { if } 1 \leqq l \leqq i, ~ \\
& =l-1 & & \text { if } i+1 \leqq l \leqq m-i-1, \\
& =l-2-\left[\frac{l+i-m}{2}\right]-\delta_{l, m+1} & & \text { if } m-i \leqq l \leqq m+1, \\
& =2 m-l-\left[\frac{l+i-m}{2}\right]+\delta_{l, m+i} & \\
& & & \\
& =2 m-\left(l+\delta_{l, m+i+1}\right. & & \text { if } m+2 \leqq l \leqq m+i+1, \\
& =2 m-(l+i) & & \text { if } m+i+2 \leqq l \leqq m+i+4 \\
& =\delta_{m, 6}+\delta_{m, 7} & & \text { and } l \leqq 2 m-i, \\
& =\delta_{m, 6} & & \text { if } m+i+5 \leqq l \leqq 2 m-i, \\
& =0 & & \text { if } l=2 m-1 \text { and } i=2,
\end{array}
$$

Consequently,

$$
\operatorname{dim} T^{1}(H)=m^{2}-(i+1) m+\frac{i(i+1)}{2}+3 \delta_{i, 2} \cdot
$$

Proposition 3.7. Suppose that $H=H_{m}-\{m+i\}$ where $H_{m}$ is ordinary and $2 i \geqq m-1 \geqq i \geqq 2$. Then 


$$
\begin{aligned}
\operatorname{dim} T^{1}(H)_{-l} & =l & & \text { if } 1 \leqq l \leqq m-i-1, \\
& =l-1-\left[\frac{l+i-m}{2}\right] & & \text { if } m-i \leqq l \leqq i, \\
& =l-2-\left[\frac{l+i-m}{2}\right]-\delta_{l, m+1} & & \text { if } i+1 \leqq l \leqq m+1, \\
& =2 m-l-\left[\frac{l+i-m}{2}\right]+\delta_{l, m+i} & & \\
& & & \text { if } m+2 \leqq l \leqq 2 m-i, \\
& =i-\left[\frac{l+i-m}{2}\right]+\delta_{l, m+i} & & \text { if } 2 m-i+1 \leqq l \leqq m+i, \\
& =1 & & \text { if } l=m+i+1, \\
& =\delta_{m, 5} & & \text { if } l=m+4 \text { and } i=2, \\
& =\delta_{m, 5}+\delta_{m, 4} & & \text { if } l=2 m \text { and } i=2, \\
& =\delta_{m, 4}+\delta_{m, 3} & & \text { if } l=2 m+2, \\
& =\delta_{m, 4}+\delta_{m, 3} & & \text { if } l=3 m \text { and } i=m-1, \\
& =\delta_{m, 3} & & \text { if } l=4 m, \\
& =0 & & \text { otherwise. }
\end{aligned}
$$

Consequently,

$$
\begin{aligned}
\operatorname{dim} T^{1}(H) & =m^{2}-(i+1) m+\frac{i(i+1)}{2}+2 \delta_{m, 4} & & \text { if } i \geqq 3, \\
& =m^{2}-3 m+5+\delta_{m, 3} & & \text { if } i=2 .
\end{aligned}
$$

Proof. We note that $2 a(H)-c(H)=m-i+1$. Hence for $1 \leqq l \leqq m-i+1$ one has $\operatorname{dim} T_{-l}=\sharp G_{-l}-1$. Also note that

$$
\begin{aligned}
G_{-l} & =\left\{a_{0}, \cdots, a_{l-1}, a_{l+i}\right\} & & \text { if } 1 \leqq l \leqq m-i-1, \\
& =\left\{a_{0}, \cdots, a_{l-1}\right\} & & \text { if } m-i \leqq l \leqq i, \\
& =\left\{a_{0}, \cdots, a_{i}, \cdots, a_{l-1}\right\} & & \text { if } i+1 \leqq l \leqq m-1, \\
& =\left\{a_{1}, \cdots, a_{m-1}\right\} & & \text { if } l=m, \\
& =S_{H}-\left\{a_{i}, a_{l-m}\right\} & & \text { if } m+1 \leqq l \leqq m+i-1, \\
& =S_{H}-\left\{a_{l-m}\right\} & & \text { if } m+i \leqq l \leqq 2 m-1, \\
& =S_{H} & & \text { if } l \geqq 2 m \text { and } l \neq 2 m+i, \\
& =S_{H}-\left\{a_{i}\right\} & & \text { if } l=2 m+i .
\end{aligned}
$$

If $m-i+2 \leqq l \leqq m+1$ then $R_{-l}=\left\{f_{j, k} \mid a_{j}+a_{k}=m+l+i\right\}=$ $\left\{f_{j, k} \mid j+k=l+i-m\right.$ and $\left.k \neq i\right\}$. Hence $\operatorname{dim} R_{-l}=[(l+i-m) / 2]-$ $\delta_{l, m+1}$.

If $m+2 \leqq l \leqq 2 m-i$ set $q=l-m$. Then

$$
\begin{aligned}
R_{-l} & =\left\{f_{j, k} \mid a_{j}+a_{k}=2 m+i+q \text { or } a_{j}+a_{k}<2 m+q\right\} \\
& =\left\{f_{j, k} \mid j+k=i+q \text { and } j, k \neq i \text { or } j+k \leqq q-1\right\} .
\end{aligned}
$$


Hence

$$
R_{-l}=\operatorname{span}\left\{f_{1, q+i-1}, \cdots, \hat{f}_{q, i}, \cdots, f_{[q+i / 2],\{q+i / 2]}, f_{1,1}, \cdots, f_{1, q-2}\right\}
$$

and $\operatorname{dim} R_{-l}=q+[(q+i) / 2]-3=l-m+[(l+i-m) / 2]-3$.

If $2 m-i+1 \leqq l \leqq m+i$ then

$$
\begin{aligned}
R_{-l} & =\left\{f_{j, k} \mid a_{j}+a_{k}=2 m+i+q \text { or } a_{j}+a_{k}<2 m+q\right\} \\
& =\left\{f_{j, k} \mid j+k=i+q \text { and } j, k \neq i \text { or } j+k \leqq q-1\right\} \\
& =\operatorname{span}\left\{f_{i+q-m+1, m-1}, \cdots, \widehat{f_{q, i},}, \cdots, f_{[(q+i) / 2],\{(q+i) / 2\}}, f_{1,1}, \cdots, f_{1, q-2}\right\} .
\end{aligned}
$$

Hence $\operatorname{dim} R_{-l}=m-1-\{(q+i) / 2\}+q-2=m+[(q+i) / 2]-i-3$.

Suppose $l=m+i+1 \geqq 2 m-i+1$ so that $2 i \geqq m$. Then if $i=m-1$ we have $l=2 m$ and $R_{-l}=\operatorname{span}\left\{f_{1,1}, \cdots, f_{1, m-2}\right\}$ so that $\operatorname{dim} T_{-l}=1$. If $i \leqq m-2$ then $R_{-l}=\operatorname{span}\left\{f_{1,1}, \cdots, f_{1, i-1}, f_{2 i+2-m, m-1}, \cdots\right.$, $\left.f_{i-1, i+2}\right\}$ and has rank $m-3$ so again $\operatorname{dim} T_{-l}=1$.

Now suppose $m+i+2 \leqq l \leqq 2 m-1$ and set $q=l-m$. If $i=2$ then $m=5$ and $R_{-l}=\left\{f_{1,1}, f_{3,3}\right\}$ so $\operatorname{dim} T_{-l}=1$. If $i \geqq 3, R_{-l}=$ $\operatorname{span}\left\{f_{1,1}, \cdots, \widehat{f_{1, i}}, \cdots, f_{1, q-2}, f_{i+q-m+1, m-1}, \cdots, f_{i-1, q+1}, f_{i+1, q-1}, f_{2, i-1}\right\}$ so that $\operatorname{dim} R_{-l}=m-2$ and $T_{-l}=0$.

Assume that $l=2 m>m+i+1$, so $i \leqq m-2$. If $i \geqq 3$ then $R_{-l}=\operatorname{span}\left\{f_{1,1}, \cdots, \widehat{f_{1, i}}, \cdots, f_{1, m-2}, f_{2, i-1}, f_{i+1, m-1}\right\}$ and $T_{-l}=0$.

If $i=2$ and $m=4$ or 5 then $R_{-l}=\left\{f_{1,1}, \widehat{f_{1,2}}, \cdots, f_{1, m-2}, f_{3, m-1}\right\}$ so that $\operatorname{dim} T_{-l}=1$.

Now suppose $l \geqq 2 m+1$ and set $q=l-[l / m] m$. If $q=1$ or $q=i$ and $l \geqq 3 m+i$ then

$$
R_{-l} \supseteqq\left\{f_{1,1}, \cdots, f_{1, m-1}\right\} \text {. }
$$

If $l=2 m+i$ so that $G_{-l}=S_{H}-\left\{a_{i}\right\}$ then $R_{-l}$ is spanned by:

$$
\begin{aligned}
& \left\{f_{1,1}, \cdots, \widehat{f_{1, i-1}}, \widehat{f_{1, i}}, \cdots, f_{1, m-1}, f_{2, i-1}\right\} \text { if } i \geqq 3, \\
& \left\{f_{1,3}, \cdots, f_{1, m-1}\right\} \text { if } i=2 \text { and } m \leqq 4, \\
& \left\{f_{1,3}, f_{1,4}, f_{3,3}\right\} \text { if } i=2 \text { and } m=5 .
\end{aligned}
$$

Consequently $\operatorname{dim} T_{-l}=\delta_{i, 2}\left(\delta_{m, 3}+\delta_{m, 4}\right)$. Suppose $q=2 \leqq i-1$. Then $R_{-l}$ is spanned by:

$$
\begin{aligned}
& \left\{f_{1,2}, \cdots, \widehat{f_{1, i}}, \cdots, f_{1, m-1}, f_{2,2}, f_{2, i-1}\right\} \text { if } i \geqq 4, \\
& \left\{f_{1,2}, \widehat{f_{1,3}}, \cdots, f_{1, m-1}, f_{2,2}, f_{2, m-1}\right\} \text { if } i=3 \text { and } m \geqq 5, \\
& \left\{f_{1,2}, f_{2,2}\right\} \text { if } i=3 \text { and } m=4 \text { and } l=2 m+2, \\
& \left\{f_{1,2}, f_{1,3}, f_{2,2}\right\} \text { if } i=3, m=4 \text { and } l \geqq 3 m+2 .
\end{aligned}
$$

We note that $\operatorname{dim} T_{-(2 m+2)}=\delta_{m, 3}+\delta_{m, 4}$. Now suppose $3 \leqq q \leqq m-1$ and that $q \neq i$. Then $R_{-l}$ is spanned by: 


$$
\begin{aligned}
& \left\{f_{1,1}, \cdots, \widehat{f_{1, q-1}}, \cdots, \widehat{f_{1, i}}, \cdots, f_{1, m-1}, \hat{f_{2, q-1}}, f_{2, i-1}\right\} \text { if } i \geqq 3 \text { and } q \neq i+1 \\
& \left\{f_{1,1}, \cdots, \widehat{f_{1,2}}, \cdots, f_{1, m-1}, f_{i+1,2+1}\right\} \text { if } q=i+1 \leqq m-1, \\
& \left\{f_{1,1}, f_{1,2}, \widehat{f_{1,3}}, f_{1,4}, f_{3,3}\right\} \text { if } i=2, m=5, q=4 .
\end{aligned}
$$

Hence $\operatorname{dim} R_{-l}=m-1$ and $T_{-l}=0$. If $q=0$ so that $l=[l / m] m \geqq$ $3 m$, then $R_{-l}$ is spanned by:

$$
\begin{aligned}
& \left\{f_{1,1}, \cdots, f_{1, m-2}, f_{i+1, m-1}\right\} \text { if } i \leqq m-2, \\
& \left\{f_{1,1}, \cdots, f_{1, m-2}\right\} \text { if } i=m-1, m \leqq 4 \text { and } l=3 m, \\
& \left\{f_{1,1}, \cdots, f_{1, m-2}, f_{3, m-2}\right\} \text { if } i=m-1 \text { and } m \geqq 5, \\
& \left\{f_{1,1}\right\} \text { if } i=m-1, m=3 \text { and } l=4 m, \\
& \left\{f_{1,1}, \cdots, f_{1, m-2}, f_{2,2}\right\} \text { if } i=m-1, m=4 \text { and } l \geqq 4 m \\
& \text { or } m=3 \text { and } l \geqq 5 m .
\end{aligned}
$$

Hence

$$
\begin{aligned}
& \operatorname{dim} T_{-3 m}=\delta_{m, 3}+\delta_{m, 4} \cdot \delta_{i, 3} \\
& \operatorname{dim} T_{-4 m}=\delta_{m, 3} \\
& \operatorname{dim} T_{-l}=0 \text { if } m \mid l \text { and } l \geqq 5 m .
\end{aligned}
$$

COROLLARY 3.8. If $H$ is negatively graded of the third type with $c(H)=m+i+1 \leqq 2 m$ then

$$
\begin{aligned}
\operatorname{dim} T^{1}(H) & =m^{2}-(i+1) m+\frac{i(i+1)}{2}+2 \delta_{m, 4} \text { if } i \geqq 3, \\
& =m^{2}-3 m+6-\delta_{m, 4}-\delta_{i k, 5} \text { if } i=2 .
\end{aligned}
$$

If $c(H)=2 m+2$ then

$$
\operatorname{dim} T^{1}(H)=\frac{(m-1) m}{2}+2+3 \delta_{m, 3}+2 \delta_{m, 4} .
$$

4. The obstruction of the formal moduli space. Let $B=B_{H}$ be negatively graded and let $T \mid S$ represent the versal deformation of $B \mid k$ in the sense of Schlessinger [6]. Then $\left(S, m_{S}\right)$ is a complete noetherian $k$-algebra with residue field $k, T$ is flat as an $S$-module and $T \otimes_{S} k \cong B$.

Pinkham [3] has shown that $T \mid S$ admit gradings as $k$-algebras which are compatible with the structure of $B$ as a graded $k$-algebra. One then has the isomorphism $T^{1}(B) \cong \operatorname{Hom}_{k}\left(m_{S} / m_{S}^{2}, k\right)$ in the category of graded $k$-vector spaces. Thus $\operatorname{dim} T^{1}(B)$ also is the dimension of the tangent space $\left(m_{S} / m_{S}^{2}\right)^{*}$ of the formal moduli space Spec $(S)$.

We say the formal moduli space is unobstructed if $S$ is a regular 
local ring. Now $S$ is regular if and only if Krull-dim $S=\operatorname{dim}\left(m_{S}\right)$ $m_{S}^{2}$ ) if and only if $S$ is formally smooth over $k$ ([2], Proposition 28. M). Thus the formal moduli space is unobstructed if and only if $\operatorname{dim} T^{1}(B)=$ Krull-dim $S$.

Let $U$ denote that open subset of $\operatorname{Spec}(S)$ cansisting of all points having smooth fibers, i.e., $U=\{x \in \operatorname{Spec}(S) \mid T(x)$ is smooth over $\kappa(x)\}$ where $T(x)=T \boldsymbol{\bigotimes}_{S} \kappa(x)$ and $\kappa(x)=A_{x} / \mathfrak{p}_{x} A_{x}$.

In [5] we showed that $U$ is nonempty (as $B$ can be smoothed) and effectively computed the dimension of $U$. We note that

$$
\operatorname{dim} U \leqq \operatorname{dim} \operatorname{Spec}(S) \leqq \operatorname{dim} T^{1}(B) .
$$

Hence $\operatorname{Spec}(S)$ is unobstructed iff $\operatorname{dim} U=\operatorname{dim} T^{1}(B)$.

We now recall the dimension formula for $U$ and compare $\operatorname{dim} U$ to $\operatorname{dim} T^{1}(B)$.

If $H$ is a numerical semigroup let End $(H)=\left\{n \in N \mid n+H^{+} \subset H\right\}$ where $H^{+}=H-\{0\}$. Let $\lambda(H)=[\operatorname{End}(H): H]$ so that $1 \leqq \lambda(H) \leqq$ $g(H)=g$.

Proposition 4.1. If $H$ is negatively graded with $\lambda(H)=\lambda$, $g(H)=g$ and $U$ is as above then

$$
\operatorname{dim} U=2 g+\lambda-1 .
$$

Proof. See [5], proof of Corollary 6.3.

Now suppose that $H$ is ordinary or hyperordinary of multiplicity $m$ with $a(H)=p m+i$ (recall that $a(H)=\inf \{H-m N\}$ ). Then $g(H)=p(m-1)+i-1$ and $\lambda(H)=m-1$ ([5], Proposition 2.2). Thus $\operatorname{dim} U=2 g+\lambda-1=(2 p+1)(m-1)+2 i-3$. Combining this with Corollary 3.4 we obtain:

Proposition 4.2. Suppose that $H$ is ordinary or hyperordinary of multiplicity $m$ with $a(H)=p m+i$. Then

$$
\begin{aligned}
\operatorname{dim} T^{1}(H)-\operatorname{dim} U & =p(m-1)(m-3)+i(i-4)+3+\delta_{i, 2} & & \text { if } m \geqq 3, \\
& =0 & & \text { if } m=2 .
\end{aligned}
$$

Consequently the formal moduli space for $B_{H}$ is unobstructed iff $m \leqq 3$.

Now suppose $H$ is negatively graded of the third type with $m(H)=m$ and $m+i$ a gap for $H$. Then $g(H)=m+\delta_{i, 1}$ and $\lambda(H)=$ $m-i-\delta_{i, 1}(m-2)$ ([5], Proposition 2.2). Hence $\operatorname{dim} U=2 g+\lambda-$ $1=3 m-i-1-\delta_{i, 1}(m-4)$. Combining this with Corollary 3.8 we obtain: 
Proposition 4.3. Suppose that $H=H_{m}-\{m+i\}$ where $H_{m}$ is ordinary and $2 \leqq i \leqq m-1$. Let $U$ be as above. Then

$$
\begin{aligned}
\operatorname{dim} T^{1}(H)-\operatorname{dim} U=(m-3)^{2}-\delta_{m, 4}-\delta_{m, 5} \quad \text { if } i=2, \\
=m^{2}-(i+4) m+\frac{(i+1)(i+2)}{2}+2 \delta_{m, 4} \quad \text { if } i \geqq 3 .
\end{aligned}
$$

If $H=H_{m}-\{m+1,2 m+1\}$ then

$$
\operatorname{dim} T^{1}(H)-\operatorname{dim} U=\frac{m(m-5)}{2}+3 \delta_{m, 3}+2 \delta_{m, 4} .
$$

Summarizing, the formal moduli space for $B_{H}$ is unobstructed iff $m \leqq 4$ or $m=5$ and $i \neq 2$ (i.e., $m+2 \in H$ ).

\section{REFERENCES}

1. S. Lichtenbaum and M. Schlessinger, The cotangent complex of a morphism, Trans. Amer. Math. Soc., 128 (1967), 41-70.

2. H. Matsumura, Commutative Algebra, Benjamin, New York, 1970.

3. H. Pinkham, Deformations of Algebraic Varieties with $\mathrm{G}_{m}$ Action, Asterique 20, Soc. Math. France, 1974.

4. D. S. Rim, Formal Deformation Theory (SGA 7-I), Séminaire de Géométrie Algébrique du Bois-Marie, 1967-1969, Lecture Notes in Mathematics, No. 288, Springer-Verlag, Berlin, 1972.

5. D. S. Rim and M. A. Vitulli, Weierstrass points and monomial curves, J. Alg., 48 (1977).

Received November 7, 1977.

UNIVERSITY OF OREGON

EugENE, OR 97403 


\section{PACIFIC JOURNAL OF MATHEMATICS}

\section{EDITORS}

DoNALD BABBITT (Managing Editor)

University of California

Los Angeles, California 90024

HUGo Rossi

University of Utah

Salt Lake City, UT 84112

C. C. MOORE and ANDREW OGG

University of California

Berkeley, CA 94720
J. DUGUNDJI

Department of Mathematics University of Southern Californı Los Angeles, California 90007

R. Finn and J. Milgram Stanford University Stanford, California 94305

\section{ASSOCIATE EDITORS}

E. F. BECKENBACH

B. H. Neumann
F. WOLF

K. YOSHIDA

\section{SUPPORTING INSTITUTIONS}

UNIVERSITY OF BRITISH COLUMBIA CALIFORNIA INSTITUTE OF TECHNOLOGY UNIVERSITY OF CALIFORNIA MONTANA STATE UNIVERSITY UNIVERSITY OF NEVADA, RENO NEW MEXICO STATE UNIVERSITY OREGON STATE UNIVERSITY UNIVERSITY OF OREGON
UNIVERSITY OF SOUTHERN CALIFORNIA STANFORD UNIVERSITY UNIVERSITY OF HAWAII UNIVERSITY OF TOKYO UNIVERSITY OF UTAH WASHINGTON STATE UNIVERSITY UNIVERSITY OF WASHINGTON 


\section{Pacific Journal of Mathematics}

Vol. 82, No. $1 \quad$ January, 1979

Werner Bäni, Subspaces of positive definite inner product spaces of countable dimension ...................................... 1

Marilyn Breen, The dimension of the kernel of a planar set..............

Kenneth Alfred Byrd, Right self-injective rings whose essential right ideals

are two-sided

Patrick Cousot and Radhia Cousot, Constructive versions of Tarski's fixed

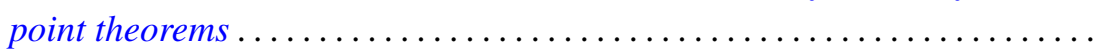

Ralph S. Freese, William A. Lampe and Walter Fuller Taylor, Congruence lattices of algebras of fixed similarity type. $I \ldots \ldots \ldots \ldots \ldots \ldots \ldots$

Cameron Gordon and Richard A. Litherland, On a theorem of Murasugi .....

Mauricio A. Gutiérrez, Concordance and homotopy. I. Fundamental

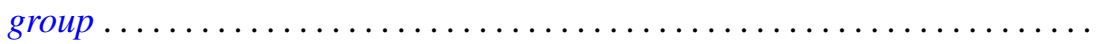

Richard I. Hartley, Metabelian representations of knot groups .............

Ted Hurley, Intersections of terms of polycentral series of free groups and free

Lie algebras ........................................

Roy Andrew Johnson, Some relationships between measures ............ 117

Oldřich Kowalski, On unitary automorphisms of solvable Lie algebras .......

Kee Yuen Lam, $K O$-equivalences and existence of nonsingular bilinear

maps...................................................

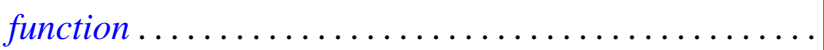

Robert A. Messer and Alden H. Wright, Embedding open 3-manifolds in compact 3-manifolds ............................

Gerald Ira Myerson, A combinatorial problem in finite fields. I . .

James Nelson, Jr. and Mohan S. Putcha, Word equations in a band of paths.

Baburao Govindrao Pachpatte and S. M. Singare, Discrete generalized Gronwall inequalities in three independent variables . .

William Lindall Paschke and Norberto Salinas, $C^{*}$-algebras associated with free products of groups ........................

Bruce Reznick, Banach spaces with polynomial norms ....

David Rusin, What is the probability that two elements of a finite group

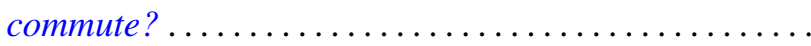

M. Shafii-Mousavi and Zbigniew Zielezny, On hypoelliptic differential operators of constant strength ...

Joseph Gail Stampfli, On selfadjoint derivation ranges .... . . .

Robert Charles Thompson, The case of equality in the matrix-valued triangle

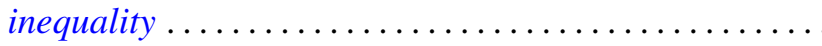

Marie Angela Vitulli, The obstruction of the formal moduli space in the negatively graded case. 